\title{
A NEW EXPONENTIALLY FITTED TRIANGULAR FINITE ELEMENT METHOD FOR THE CONTINUITY EQUATIONS IN THE DRIFT-DIFFUSION MODEL OF SEMICONDUCTOR DEVICES
}

\author{
SONG WANG ${ }^{1}$
}

\begin{abstract}
In this paper we present a novel exponentially fitted finite element method with triangular elements for the decoupled continuity equations in the drift-diffusion model of semiconductor devices. The continuous problem is first formulated as a variational problem using a weighted inner product. A Bubnov-Galerkin finite element method with a set of piecewise exponential basis functions is then proposed. The method is shown to be stable and can be regarded as an extension to two dimensions of the well-known Scharfetter-Gummel method. Error estimates for the approximate solution and its associated flux are given. These $h$-order error bounds depend on some first-order seminorms of the exact solution, the exact flux and the coefficient function of the convection terms. A method is also proposed for the evaluation of terminal currents and it is shown that the computed terminal currents are convergent and conservative.
\end{abstract}

Résumé. Dans cet article nous présentons une méthode d'éléments finis avec éléments triangulaires et adaptation exponentielle pour les équations de continuité découplées dans le modèle de convectiondiffusion des semi-conducteurs.

AMS Subject Classification. Primary 65N30, 65P05.

Received: November 29, 1995. Revised: April 2, 1997 and November 25, 1997.

\section{INTRODUCTION}

Solutions of the semiconductor device equations display sharp interior layers due to the abrupt change in doping profile. Applications of classical discretisation methods such as the central difference or the standard piecewise linear finite element method to these equations often yield results with non-physical properties such as spurious oscillations, unless impractically fine meshes are used. To overcome such difficulties Scharfetter and Gummel [14] proposed a method in the one dimensional space, known as the Scharfetter-Gummel method. The same idea was also proposed independently by Allen and Southwell [1]. Extensions of the Scharfetter-Gummel method to higher dimensions have been presented by different authors ( $c f$., for examples, [2-4,6, 8-13]). One of these is the Scharfetter-Gummel box method ( $c f$. , for example, $[2,4,9,13])$ which has been widely used in practice. This method is based on Delaunay triangulations and on the one-dimensional Scharfetter-Gummel approximation to the flux on each edge of a mesh. Recently Miller and Wang [11] present an analysis for this method using a Petrov-Galerkin finite element formulation. In their analysis the basis functions for the finite element trial space are determined by analytically solving a two-point boundary value problem on each edge. An error estimate for the Slotboom variables (cf. [16]) is given and the computed terminal currents are proved to be convergent and conservative. However this error estimate is still not satisfactory, because it depends exponentially on the maximum or minimum value of the electro-static potential which may be

Key words and phrases. exponential fitting, finite element method, semiconductors.

${ }^{1}$ School of Mathematics and Statistics Curtin University of Technology, Perth 6845, Australia. e-mail: swang@cs.curtin.edu.au (c) EDP Sciences, SMAI 1999 
very large in practice. Also the Slotboom variables depend exponentially on the electro-static potential, and thus they are physically less interesting than electron and hole concentrations. These problems also appear in $[8,10,12,13]$. In [3] Brezzi, Marini and Pietra present some two-dimensional extensions of the ScharfetterGummel method based on a mixed formulation and a hybrid formulation. Error estimates for the approximate fluxes are presented, but no error estimate is given for the approximate electron and hole concentrations.

In this paper we propose a new finite element method for the semiconductor continuity equations, based on a Bubnov-Galerkin formulation. Although the method is presented for triangular meshes, it can be easily extended to tetrahedral meshes in three dimensions. The continuous problem is first formulated as a weak one using a weighted inner product. The weak problem is then approximated by a Bubnov-Galerkin finite element method based on a set of special basis functions. Each of these basis functions and the auxiliary flux associated with it are uniquely determined by a $3 \times 3$ linear system. The basis functions are piecewise exponential, and the method can be regarded as an extension of the Scharfetter-Gummel scheme to higher dimensions. This method is proved to be stable, and the errors in a weighted energy norm for the approximate electron and hole concentrations are shown to be of $O(h)$ order. A method is proposed for the evaluation of terminal currents and the computed terminal currents are shown to be convergent and conservative. This paper is organised as follows.

In the next section we give a mathematical description of the semiconductor device problem. The continuous equations are formulated as a variational problem using a weighted inner product. It is shown that this weak problem has a unique solution. In Section 3 we present a finite element method based on the Bubnov-Galerkin formulation. The basis functions for the trial and test spaces are defined by solving a set of local two point boundary problems with constant coefficients in each element. In Section 4 the method is proved to be stable with respect to an weighted energy norm, and $O(h)$ error estimates for electron and hole concentrations and their associated fluxes in proper norms are given which differ from results for conventional methods in the way that they depend only on first order seminorms of the exact solutions, the exact fluxes and the coefficient functions of the convection terms. Physically these exact fluxes are well behaved although it is not mathematically provable. Unlike those in $[8,10,11]$ the arbitrary constants in the error bounds do not depend exponentially on the maximum or minimum value of the electro-static potential, though the weighted norms depend exponentially on the electro-static potential. In Section 5 we propose a method to evaluate the terminal currents. It is shown that the computed terminal currents are convergent and conservative.

\section{The CONTINUOUS PROBLEM}

The stationary behaviour of semiconductor devices can be described by the following (scaled) nonlinear system of second-order elliptic equations [18]

$$
\begin{aligned}
& \nabla^{2} \psi-n+p=-D, \\
& \nabla \cdot \boldsymbol{J}_{n}-R(\psi, n, p)=0, \\
& \nabla \cdot \boldsymbol{J}_{p}+R(\psi, n, p)=0,
\end{aligned}
$$

with appropriate boundary conditions, where

$$
\begin{aligned}
& \boldsymbol{J}_{n}=\nabla n-n \nabla \psi, \\
& \boldsymbol{J}_{p}=-(\nabla p+p \nabla \psi) .
\end{aligned}
$$

Here $\psi$ is the electrostatic potential, $n$ is the electron concentration, $p$ is the hole concentration, $D$ denotes the doping function and $R$ denotes the recombination/generation rate which is assumed to be monotone with respect to $n$ and $p$, i.e.

$$
\frac{\partial R}{\partial n} \geq 0, \frac{\partial R}{\partial p} \geq 0 .
$$


Using Gummel's method [7] and Newton's method we can decouple and linearise the above system so that at each iteration step we sequentially solve a Poisson equation and two continuity equations. We assume that the Dirichlet boundary conditions for $\psi, n$ and $p$ are homogeneous. The inhomogeneous case can be transformed into the homogeneous case by subtracting a special function satisfying the boundary conditions. Thus the decoupled continuity equations are of the following form

$$
\begin{aligned}
-\nabla \cdot \boldsymbol{f}_{u, \boldsymbol{a}}+G u & =F, \text { in } \Omega \\
\boldsymbol{f}_{u, \boldsymbol{a}} & =\nabla u-\boldsymbol{a} u, \\
\left.u\right|_{\partial \Omega_{D}} & =0,\left.\boldsymbol{f}_{u, \boldsymbol{a}} \cdot \boldsymbol{n}\right|_{\partial \Omega_{N}}=0,
\end{aligned}
$$

where $\Omega \in \mathbb{R}^{2}, \partial \Omega=\partial \Omega_{D} \cup \partial \Omega_{N}$ is the boundary of $\Omega, \partial \Omega_{D} \cap \partial \Omega_{N}=\emptyset$ and $\boldsymbol{n}$ denotes the unit outward normal vector on $\partial \Omega$. The vector-valued function $\boldsymbol{a}$ is $\nabla \psi$ and $-\nabla \psi$ respectively for the electron and hole concentrations, and the flux $\boldsymbol{f}_{u, \boldsymbol{a}}$ is the electron or hole current. We only consider the case that $\boldsymbol{a}=\nabla \psi$. Results for hole concentration follow immediately on replacing $\psi$ by $-\psi$.

In what follows $L^{p}(S)$ and $W^{m, p}(S)$ denote the usual Sobolev spaces with norms $\|\cdot\|_{p, S}$ and $\|\cdot\|_{m, p, S}$, respectively, for any measurable open set $S \subset \mathbb{R}^{n}(n=1,2)$ and any $1 \leq p \leq \infty$. The inner product on $L^{2}(S)$ and $\left(L^{2}(S)\right)^{2}$ is denoted by $(\cdot, \cdot)_{S}$ and the kth order seminorm on $W^{m, p}(S)$ by $|\cdot|_{k, p, S}$. The Sobolev space $W^{m, 2}(S)$ is written $H^{m}(S)$ with corresponding norm and $k$ th order seminorm $\|\cdot\|_{m, S}$ and $|\cdot|_{k, S}$, respectively. When $S=\Omega$, we omit the subscript $S$ in the above notation. We put $\boldsymbol{L}^{2}(\Omega)=\left(L^{2}(\Omega)\right)^{2}$ and $H_{D}^{1}(\Omega)=\{v \in$ $\left.H^{1}(\Omega):\left.v\right|_{\partial \Omega_{D}}=0\right\}$. The set of continuous function on $\bar{\Omega}$ is denoted by $C^{0}(\bar{\Omega})$. We use $|\cdot|$ to denote absolute value, Euclidean length, or area depending on the context.

For the coefficient functions we assume that $G \in L^{\infty}(\Omega), G \geq 0$ for all $x \in \Omega$ and $F \in L^{2}(\Omega)$.

Before further consideration we first define a weighted inner product $(\cdot, \cdot)_{\psi}$ on $L^{2}(\Omega)$ and on $\boldsymbol{L}^{2}(\Omega)$ by

$$
(v, w)_{\psi}=\left(e^{-\psi} v, w\right)
$$

The $L^{2}$ - or $\boldsymbol{L}^{2}$-norm corresponding to this weighted inner product is denoted by $\|\cdot\|_{0, \psi}$. Using this inner product we define the following variational problem corresponding to (2.4-2.6).

Problem 2.1: Find $u \in H_{D}^{1}(\Omega)$ such that for all $v \in H_{D}^{1}(\Omega)$

$$
A_{\psi}(u, v)=(F, v)_{\psi}
$$

where $A_{\psi}(\cdot, \cdot)$ is a bilinear form on $\left(H_{D}^{1}(\Omega)\right)^{2}$ defined by

$$
A_{\psi}(u, v)=(\nabla u-\boldsymbol{a} u, \nabla v-\boldsymbol{a} v)_{\psi}+(G u, v)_{\psi} .
$$

Define a functional $\|\cdot\|_{1, \psi}$ on $H_{D}^{1}(\Omega)$ by

$$
\|v\|_{1, \psi}^{2}=A_{\psi}(v, v)
$$

Then we have the following theorem.

Theorem 2.1. The functional $\|\cdot\|_{1, \psi}$ is a norm on $H_{D}^{1}(\Omega)$.

Proof. To prove that $\|\cdot\|_{1, \psi}$ is a norm we need to show that, for any $u, v \in H_{D}^{1}(\Omega)$,

(i) $\|\lambda u\|_{1, \psi}=|\lambda|\|u\|_{1, \psi}$ for any $\lambda \in \mathbb{R}$

(ii) $\|u\|_{1, \psi} \geq 0$ and $\|u\|_{1, \psi}=0$ implies $u=0$,

(iii) $\|u-v\|_{1, \psi} \leq\|u\|_{1, \psi}+\|v\|_{1, \psi}$.

From (2.10) and (2.9) it is easy to show that (i) holds. We now prove (ii). For any $u \in H_{D}^{1}(\Omega)$, from $(2.9)$ and (2.10) we have

$$
\|u\|_{1, \psi}^{2}=\left\|\boldsymbol{f}_{u, \boldsymbol{a}}\right\|_{0, \psi}^{2}+(G u, u)_{\psi} .
$$


Therefore $\|u\|_{1, \psi} \geq 0$. When $\|u\|_{1, \psi}=0$, from (2.11) we see that

$$
\boldsymbol{f}_{u, \boldsymbol{a}} \equiv \nabla u-\boldsymbol{a} u=0 \quad \forall x \in \Omega
$$

since $G \geq 0$. The solution to the above equation is either $u=0$ or $u=e^{\psi+C}$ with $C$ an arbitrary additive constant. However, since $e^{\psi+C} \notin H_{D}^{1}(\Omega)$ we have $u=0$, proving (ii).

The proof of the triangular inequality (iii) is trivial because $\|\cdot\|_{1, \psi}^{2}$ can be expressed as the sum of two weighted inner products, as is given in (2.11).

The norm defined in (2.10) is a weighted energy norm. Using this norm we have the following theorem.

Theorem 2.2. There exists a unique solution to Problem 2.1.

Proof. To prove this theorem it suffices to show that the bilinear form $A_{\psi}(\cdot, \cdot)$ is coercive, i.e., there exists a constant $C>0$ such that for all $u \in H_{D}^{1}(\Omega)$

$$
A_{\psi}(u, u) \geq C\|u\|_{1, \psi}^{2}
$$

In fact, the above follows from (2.9) and (2.11) with $C=1$.

We comment that Theorems 2.1 and 2.2 hold for any $\psi \in H^{1}(\Omega)$. Thus, for most of our discussions below, we shall replace $\psi$ by its piecewise linear interpolant which is also in $H^{1}(\Omega)$.

\section{The Finite Element Method}

In this section we propose a novel Galerkin finite element method for Problem 2.1. For simplicity we assume hereafter that $\partial \Omega$ is polygonal.

Let $\left\{T_{h}\right\}_{h}$ be a regular sequence of meshes of $\Omega$, each $T_{h}$ consisting of triangles having diameters less than or equal to $h$. The set of vertices of $T_{h}$ is denoted $\left\{x_{i}\right\}_{1}^{N}$. Without loss of generality we assume that the nodes of $T_{h}$ are numbered such that $\left\{x_{i}\right\}_{1}^{N^{\prime}}$ is the set of mesh nodes not on $\partial \Omega_{D}$. Before further discussion, we let $\psi^{I}$ denote the interpolant of $\psi$ in the conventional piecewise linear finite element space constructed on $T_{h}$. We also let $\boldsymbol{a}^{I}=\nabla \psi^{I}$ which is constant on each triangle in $T_{h}$. In practice $\psi^{I}$ may be the numerical solution of (2.1) by a numerical method.

Corresponding to the mesh $T_{h}$, we now construct a space $S_{h} \subset H_{D}^{1}(\Omega)$ of dimension $N^{\prime}$ using the basis functions $\left\{\phi_{i}\right\}_{1}^{N^{\prime}}$ defined below. These basis functions are motivated by the idea proposed by Sever [15]. Let $t \in T_{h}$ be a triangle with vertices $x_{i}, x_{j}$ and $x_{k}$. We define a local function $\phi_{i}$ on $t$ associated with $x_{i}$ as follows. For any point $x \in t$ we use $l_{m}(m=i, j, k)$ to denote the segment connecting $x_{m}$ and $x$, and use $\boldsymbol{e}_{m}:=\left(e_{m, 1}, e_{m, 2}\right)(m=i, j, k)$ to denote the unit vector from $x_{m}$ to $x$ ( $c f$. Fig. 3.1). We now consider the following two-point boundary problem:

$$
\begin{gathered}
\frac{d}{d \boldsymbol{e}_{m}}\left(\boldsymbol{p}_{i} \cdot \boldsymbol{e}_{m}\right):=\frac{d}{d \boldsymbol{e}_{m}}\left(\frac{d g_{i}(z)}{d \boldsymbol{e}_{m}}-\boldsymbol{a}^{I} \cdot \boldsymbol{e}_{m} g_{i}(z)\right)=0 \quad z \in l_{m} \\
g_{i}\left(x_{m}\right)=\delta_{i m}, \quad g_{i}(x)=\phi_{i}(x)
\end{gathered}
$$

for $m=i, j, k$, (i.e. $\boldsymbol{p}_{i} \cdot \boldsymbol{e}_{m}$ is constant on $l_{m}$ ), where $\delta_{i m}$ denotes the Kronecker delta and $\phi_{i}(x)$ is yet to be determined. Solving (3.1) with the boundary conditions analytically yields

$$
\boldsymbol{p}_{i} \cdot \boldsymbol{e}_{m}=\frac{1}{\left|l_{m}\right|}\left[B\left(a_{m}\left|l_{m}\right|\right) \phi_{i}(x)-B\left(-a_{m}\left|l_{m}\right|\right) \delta_{i m}\right], \quad \forall x \in l_{m}, \quad m=i, j, k
$$




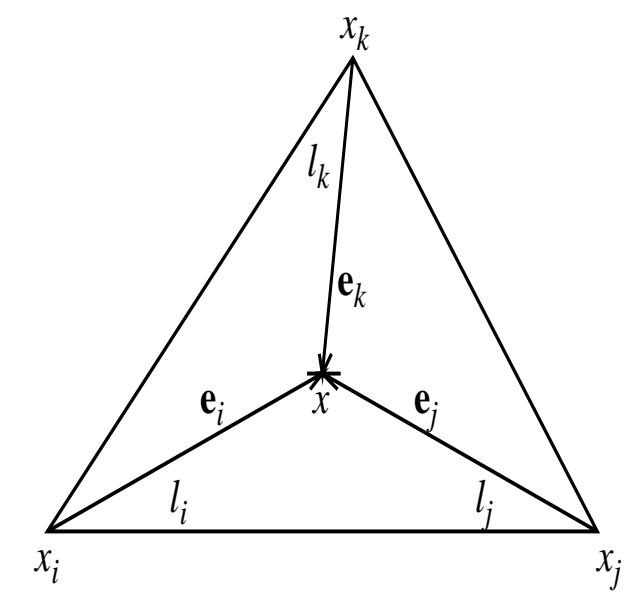

FigURE 3.1. Notation associated with the triangle $t$.

where $a_{m}=\left.\boldsymbol{a}^{I} \cdot \boldsymbol{e}_{m}\right|_{t}$ and $B(z)$ denotes the Bernoulli function defined by

$$
B(z)= \begin{cases}\frac{z}{e^{z}-1} & z \neq 0 \\ 1 & z=0\end{cases}
$$

Equation (3.2) motivates us to define the following problem.

Problem 3.1: Find $\phi_{i}$ and $\boldsymbol{p}_{i}=\left(p_{i, 1}, p_{i, 2}\right)$ such that for all $x \in \bar{t}$

$$
D(x)\left(\begin{array}{c}
p_{i, 1} \\
p_{i, 2} \\
\phi_{i}
\end{array}\right)=\left(\begin{array}{c}
-B\left(-a_{i}\left|l_{i}\right|\right) \\
0 \\
0
\end{array}\right)
$$

where $D(x)$ is a $3 \times 3$ matrix defined by

$$
D(x)=\left(\begin{array}{lll}
\left|l_{i}\right| e_{i, 1} & \left|l_{i}\right| e_{i, 2} & -B\left(a_{i}\left|l_{i}\right|\right) \\
\left|l_{j}\right| e_{j, 1} & \left|l_{j}\right| e_{j, 2} & -B\left(a_{j}\left|l_{j}\right|\right) \\
\left|l_{k}\right| e_{i, 1} & \left|l_{k}\right| e_{k, 2} & -B\left(a_{k}\left|l_{k}\right|\right)
\end{array}\right) .
$$

Any solution to Problem 3.1 defines the point values of the function $\phi_{i}$ and an auxiliary flux $\boldsymbol{p}_{i}$ for any $x \in \bar{t}$.

Similarly we can define functions $\phi_{j}$ and $\phi_{k}$ associated with $x_{j}$ and $x_{k}$ respectively. The following theorem shows that Problem 3.1 is uniquely solvable for all $x \in \bar{t}$, and that $\phi_{i}, \phi_{j}$ and $\phi_{k}$ form a system of local basis functions.

Theorem 3.1. Let $t \in T_{h}$. Then, for any $x \in \bar{t}$, there exists a unique solution to Problem 3.1. Furthermore, we have

$$
\begin{aligned}
& \phi_{i}\left(x_{i}\right)=1, \quad \phi_{i}=0 \quad \forall x \in \bar{x}_{j} x_{k}, \\
& \phi_{i}+\phi_{i}+\phi_{k}=1, \quad \boldsymbol{p}_{i}+\boldsymbol{p}_{j}+\boldsymbol{p}_{k}=-\boldsymbol{a}^{I} \quad \forall x \in \bar{t},
\end{aligned}
$$

where ${\overline{x_{j}}}_{k}$ denotes the edge of $t$ connecting $x_{j}$ and $x_{k}$. 
Proof. To prove that Problem 3.1 is uniquely solvable we need only to show that for any $x \in \bar{t}$ the system matrix $D(x)$ is non-singular, or $\operatorname{det} D(x) \neq 0$. From (3.5) we have, by direct computation,

$$
\begin{aligned}
\operatorname{det} D(x)= & \left|l_{i}\right|\left|l_{j}\right| B\left(a_{k}\left|l_{k}\right|\right)\left(e_{j, 1} e_{i, 2}-e_{j, 2} e_{i, 1}\right)+\left|l_{j}\right|\left|l_{k}\right| B\left(a_{i}\left|l_{i}\right|\right)\left(e_{k, 1} e_{j, 2}-e_{k, 2} e_{j, 1}\right) \\
& +\left|l_{k}\right|\left|l_{i}\right| B\left(a_{j}\left|l_{j}\right|\right)\left(e_{i, 1} e_{k, 2}-e_{i, 2} e_{k, 1}\right) \\
= & -\left[\left|l_{i}\right|\left|l_{j}\right| B\left(a_{k}\left|l_{k}\right|\right) \boldsymbol{e}_{z} \cdot\left(\boldsymbol{e}_{i} \times \boldsymbol{e}_{j}\right)+\left|l_{j}\right|\left|l_{k}\right| B\left(a_{i}\left|l_{i}\right|\right) \boldsymbol{e}_{z} \cdot\left(\boldsymbol{e}_{j} \times \boldsymbol{e}_{k}\right)\right. \\
& \left.+\left|l_{k}\right|\left|l_{i}\right| B\left(a_{j}\left|l_{j}\right|\right) \boldsymbol{e}_{z} \cdot\left(\boldsymbol{e}_{k} \times \boldsymbol{e}_{i}\right)\right]
\end{aligned}
$$

with $\boldsymbol{e}_{z}=(0,0,1)$ the unit vector perpendicular to $t$. From the orientations of $\boldsymbol{e}_{i}, \boldsymbol{e}_{j}, \boldsymbol{e}_{k}$ and $\boldsymbol{e}_{z}$ (cf. Fig. 3.1) we see that $\boldsymbol{e}_{z} \cdot\left(\boldsymbol{e}_{i} \times \boldsymbol{e}_{j}\right), \boldsymbol{e}_{z} \cdot\left(\boldsymbol{e}_{j} \times \boldsymbol{e}_{k}\right)$ and $\boldsymbol{e}_{z} \cdot\left(\boldsymbol{e}_{k} \times \boldsymbol{e}_{i}\right)$ are all nonnegative, and at least two of them are bounded below. Furthermore, since $B(\cdot)$ is always positive and at least two of $\left|l_{i}\right|,\left|l_{j}\right|$ and $\left|l_{k}\right|$ are not zero, we have $\operatorname{det} D(x) \neq 0$.

We now prove (3.6). When $x=x_{i}$ we have $\left|l_{i}\right|=0$, and thus from the first equation in (3.4) we obtain $\phi_{i}\left(x_{i}\right)=1$. When $x \in \bar{x}_{j} x_{k}$ we show that the solution to Problem 3.1 is $\phi_{i}(x)=0$ and $\left(p_{i, 1}, p_{i, 2}\right)$ satisfying

$$
\left(\begin{array}{cc}
e_{i, 1} & e_{i, 2} \\
e_{j, 1} & e_{j, 2}
\end{array}\right)\left(\begin{array}{c}
p_{i, 1} \\
p_{i, 2}
\end{array}\right)=\left(\begin{array}{c}
-\frac{1}{\left|l_{i}\right|} B\left(-a_{i}\left|l_{i}\right|\right) \\
0
\end{array}\right) .
$$

This $2 \times 2$ linear system is uniquely solvable because $\boldsymbol{e}_{i}$ and $\boldsymbol{e}_{j}$ are not co-linear. Since $x \in \bar{x}_{j} x_{k}$ we have $\boldsymbol{e}_{k}=-\boldsymbol{e}_{j}$. So, subtracting the third equation from the second one in (3.4) we obtain

$$
\left(\left|l_{j}\right|+\left|l_{k}\right|\right)\left(e_{j, 1} p_{i, 1}+e_{j, 2} p_{i, 2}\right)=0 .
$$

Combining this equation with the first one in (3.4) with $\phi_{i}=0$ we obtain (3.9).

Now we prove (3.7). Let $\phi=\phi_{i}+\phi_{j}+\phi_{k}$ and $\left(p_{1}, p_{2}\right):=\boldsymbol{p}=\boldsymbol{p}_{i}+\boldsymbol{p}_{j}+\boldsymbol{p}_{k}$. Since $\phi_{m}$ and $\boldsymbol{f}_{m}$ satisfy (3.4) for $m=i, j, k$, summing the three linear systems yields

$$
D(x)\left(\begin{array}{c}
p_{1} \\
p_{2} \\
\phi
\end{array}\right)=\left(\begin{array}{c}
-B\left(-a_{i}\left|l_{i}\right|\right) \\
-B\left(-a_{j}\left|l_{j}\right|\right) \\
-B\left(-a_{k}\left|l_{k}\right|\right)
\end{array}\right)
$$

with $D(x)$ defined by (3.5). We now verify that $\phi=1$ and $\boldsymbol{p}=-\boldsymbol{a}^{I}$ satisfy the above linear system. From (3.3) it is easy to verify that $B(-z)=e^{z} B(z)$. Thus, substituting $\phi=1$ into the above linear system we have, for $m=i, j, k$,

$$
\begin{aligned}
\left|l_{m}\right| \boldsymbol{e}_{m} \cdot \boldsymbol{p} & =B\left(a_{m}\left|l_{m}\right|\right)-B\left(-a_{m}\left|l_{m}\right|\right) \\
& =B\left(a_{m}\left|l_{m}\right|\right)-e^{a_{m}\left|l_{m}\right|} B\left(a_{m}\left|l_{m}\right|\right) \\
& =-\left|l_{m}\right| \boldsymbol{e}_{m} \cdot \boldsymbol{a}^{I} .
\end{aligned}
$$

Therefore we have $\boldsymbol{p}=-\boldsymbol{a}^{I}$, proving (3.7).

For each triangle having $x_{i}$ as a vertex we have defined a local function $\phi_{i}$ and an auxiliary flux $\boldsymbol{p}_{i}$ associated with $\phi_{i}$ as above. Using the Cramer's rule we can obtain the following explicit expression for $\phi_{i}(x)$ on $t$ :

$$
\phi_{i}(x)=\frac{1}{\operatorname{det} D} \cdot B\left(-a_{i}\left|l_{i}\right|\right)\left|l_{j}\right|\left|l_{k}\right|\left(e_{j, 1} e_{k, 2}-e_{j, 2} e_{i, 1}\right),
$$



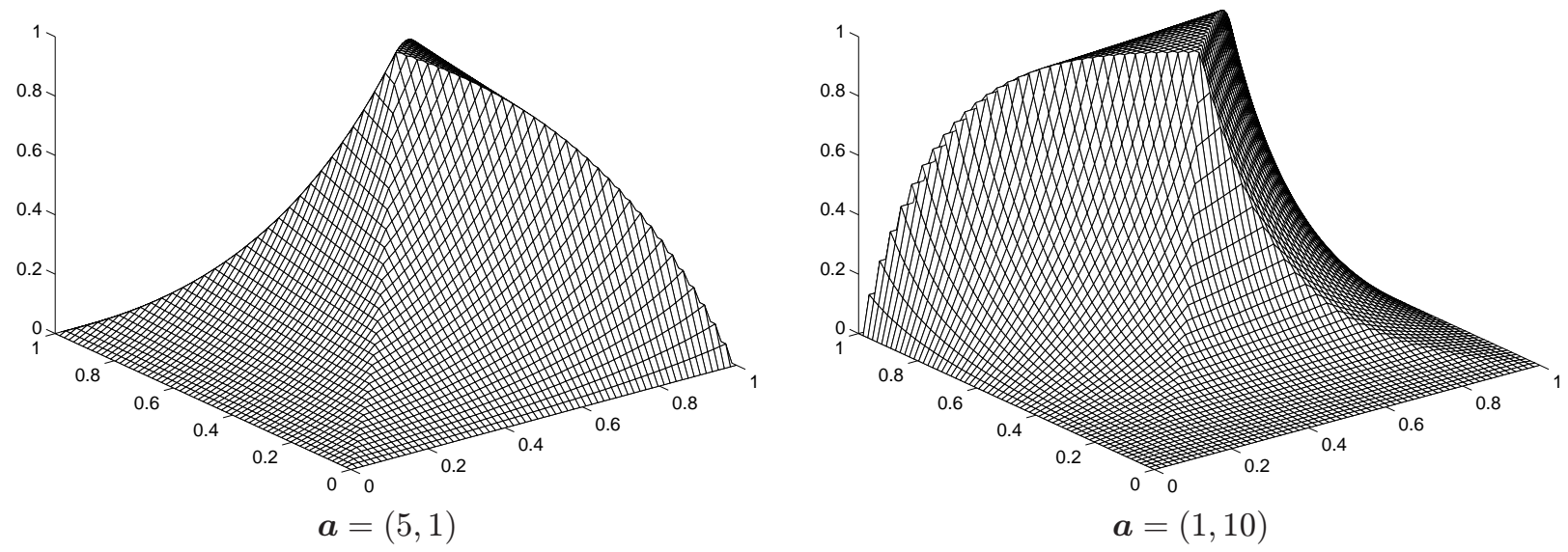

FiguRE 3.2. Hat functions for different values of $\boldsymbol{a}$.

where $\operatorname{det} D$ denotes the determinant of $D(x)$ given in (3.8). Combining all the local functions associated with $x_{i}$ we obtain a hat function $\phi_{i}$ defined on the union of all the triangles sharing $x_{i}$, denoted by $\Omega_{i}$. From Theorem 3.1 we see that this $\phi_{i}$ is unity at $x_{i}$ and 0 on $\partial \Omega_{i}$. This hat function $\phi_{i}$ can then be extended to $\Omega$ by defining $\phi_{i}(x)=0$ for all $x \in \Omega \backslash \Omega_{i}$. If we can show that $\phi_{i}$ is continuous across inter-element boundaries in $\Omega_{i}$, then $\phi_{i} \in C^{0}(\bar{\Omega}) \cap H_{D}^{1}(\Omega)$. Let $t_{1}$ and $t_{2}$ be two triangles having the edge ${\overline{x_{i} x_{j}}}_{j}$ in common, and let $\phi_{i, 1}$ and $\phi_{i, 2}$ are the local functions on $t_{1}$ and $t_{2}$ respectively defined by (3.4). When $x \in \bar{x}_{i} x_{j}$ we have that $\left(e_{j, 1}, e_{j, 2}\right)=-\left(e_{i, 1}, e_{i, 2}\right)$ (cf. Fig. 3.1). Thus multiplying the first equation in (3.4) by $\left|l_{j}\right|$ and the second equation by $\left|l_{i}\right|$, and adding the resulting equations together we have

$$
-\left[\left|l_{j}\right| B\left(a_{i}\left|l_{i}\right|\right)+\left|l_{i}\right| B\left(a_{j}\left|l_{j}\right|\right)\right] \phi_{i, m}=-\left|l_{j}\right| B\left(-a_{i}\left|l_{i}\right|\right)
$$

for $m=1,2$. So both $\phi_{i, 1}$ and $\phi_{i, 2}$ on $\bar{x}_{i} x_{j}$ are determined by the above equation, and thus $\phi_{i}$ is continuous across $\bar{x}_{i} x_{j}$. We comment that the continuity of the basis function $\phi_{i}$ does not depend on the continuity of $\boldsymbol{a}^{I}$, but depends on the continuity of the tangent component $a_{i}=\boldsymbol{a}^{I} \cdot \boldsymbol{e}_{i}$ of $\boldsymbol{a}^{I}$ along each edge having $x_{i}$ as a vertex. Although $\boldsymbol{a}^{I}$ is not continuous across element edges, its tangent derivative along each edge is continuous. In fact, the limits of $a_{i}$ as $x$ approaches $\bar{x}_{i} x_{j}$ from both sides of the edge are always $\left(\psi\left(x_{j}\right)-\psi\left(x_{i}\right)\right) /\left|\bar{x}_{i} x_{j}\right|$. To visualise this kind of hat functions, we divide $[0,1] \times[0,1]$ into four triangles by the two diagonals of this square and solve (3.4) on these triangles. The computed hat functions associated with the mid-point of the square corresponding to $\boldsymbol{a}=(5,1)$ and $\boldsymbol{a}=(1,10)$ are shown in Figure 3.2. From this we see that the hat functions are 1 at the mid-point and zero along the boundary. They are also continuous across the inter-element boundaries.

We remark that when $\boldsymbol{a}^{I} \equiv 0$, the basis function $\phi_{i}$ reduces to the standard piecewise linear basis function. On any $t \in T_{h}$, the basis function defined above satisfies a two point boundary value problem on all segments having one of the vertices of $t$ as an end-point, while the basis function defined in [11] satisfies a two point boundary value problem only on each of the edges of $t$. Therefore this is an extension of the exponential basis function defined in [11]. One visible extension of the basis function defined above is to look for $\phi_{i}$ satisfying $\nabla \cdot\left(\nabla \phi_{i}-\boldsymbol{a}^{I} \phi_{i}\right)=0$ in each $t \in T_{h}$ with appropriate Dirichlet boundary conditions on $\partial t$. However this is impractical because solving this local problem is as difficult as solving (2.4-2.6).

We comment that although (3.4) defines an auxiliary flux $\boldsymbol{p}_{i}$, we do not, in general, have $\boldsymbol{p}_{i} \equiv \nabla \phi_{i}-\boldsymbol{a}^{I} \phi_{i}=$ : $\boldsymbol{f}_{\phi_{i}, \boldsymbol{a}^{I}}$ on a triangle $t$ having $x_{i}$ as one vertex. Nevertheless, the following theorem shows that $\boldsymbol{p}_{i}=\boldsymbol{f}_{\phi_{i}, \boldsymbol{a}^{I}}$ at the three vertices of $t$. 
Theorem 3.2. For any triangle t having vertices $x_{i}, x_{j}$ and $x_{k}$, let $\phi_{i}$ and $\boldsymbol{p}_{i}$ be the solution to (3.4) and $\boldsymbol{f}_{\phi_{i}, \boldsymbol{a}^{I}}$ be the flux associated with $\phi_{i}$. Then we have

$$
\boldsymbol{f}_{\phi_{i}, \boldsymbol{a}^{I}}\left(x_{m}\right)=\boldsymbol{p}_{i}\left(x_{m}\right) \quad \text { for } \quad m=i, j, k .
$$

Proof. We first prove the case that $m=i$ in (3.11). For any $x \in t$, we parametrize the half line starting from $x_{i}$ passing though $x$ by $s$ so that $s=0$ corresponds to $x=x_{i}$ and $s=\left|l_{i}\right|$ corresponds to $x=x_{i}$. Thus, the first equation in (3.4) can be rewritten as

$$
p_{i}(s):=\boldsymbol{p}_{i}(s) \cdot \boldsymbol{e}_{i}=\frac{1}{s}\left[B\left(a_{i} s\right) \phi_{i}(s)-B\left(-a_{i} s\right)\right] \quad s>0,
$$

where $a_{i}=\boldsymbol{a}^{I} \cdot \boldsymbol{e}_{i}$. We assume that $a_{i} \neq 0$. When $a_{i}=0$ this equation reduces to a simpler one. Now, from this equation and (3.3) we get

$$
\phi_{i}(s)=\frac{\left(e^{a_{i} s}-1\right) p_{i}(s)}{a_{i}}-\frac{e^{a_{i} s}-1}{e^{-a_{i} s}-1} .
$$

Differentiating both sides of the above with respect to $s$ we get

$$
\begin{aligned}
\phi_{i}^{\prime} & =\frac{p_{i}}{a_{i}} e^{a_{i} s} a_{i}+\left(e^{a_{i} s}-1\right) \frac{p_{i}^{\prime}}{a_{i}}-a_{i} \frac{2-e^{a_{i} s}-e^{-a_{i} s}}{\left(e^{-a_{i} s}-1\right)^{2}} \\
& =p_{i} e^{a_{i} s}+\left(e^{a_{i} s}-1\right) \frac{p_{i}^{\prime}}{a_{i}}-a_{i} \frac{e^{a_{i} s}-1}{e^{-a_{i} s}-1} .
\end{aligned}
$$

Thus from (3.12) and this we obtain

$$
\phi_{i}^{\prime}-a_{i} \phi_{i}=p_{i}+\left(e^{a_{i} s}-1\right) \frac{p_{i}^{\prime}}{a_{i}}
$$

Therefore, letting $s \rightarrow 0^{+}$on both sides of the above we have

$$
\left(\phi_{i}^{\prime}-a_{i} \phi_{i}\right)(0)=p_{i}(0) .
$$

Note that (3.13) is in the parametric form. It is equivalent to

$$
\left(\boldsymbol{f}_{\phi_{i}, \boldsymbol{a}^{I}} \cdot \boldsymbol{e}_{i}\right)\left(x_{i}\right)=\left(\frac{d \phi_{i}}{d \boldsymbol{e}_{i}}-\boldsymbol{a}^{I} \cdot \boldsymbol{e}_{i} \phi_{i}\right)\left(x_{i}\right)=\boldsymbol{p}\left(x_{i}\right) \cdot \boldsymbol{e}_{i} .
$$

Since $\boldsymbol{e}_{i}$ is the direction from $x_{i}$ to any point $x \in t$, (3.11) with $m=i$ follows from the above equality.

The proofs for the cases that $m=j$ and $k$ in (3.11) are the same as above, because the last two equations in (3.4) are special cases of the first equation in (3.4) (with the last term in (3.12) replaced by 0). Thus we have proved the theorem.

We now put $S_{h}=\operatorname{span}\left\{\phi_{i}\right\}_{1}^{N^{\prime}}$. From the above discussion we see $S_{h} \subset C^{0}(\bar{\Omega}) \cap H_{D}^{1}(\Omega)$. Using the finite element space $S_{h}$ we define the following Bubnov-Galerkin problem.

Problem 3.2: Find $u_{h} \in S_{h}$ such that for all $v \in S_{h}$

$$
A_{\psi^{I}}\left(u_{h}, v_{h}\right)=\left(F, v_{h}\right)_{\psi^{I}}
$$

where $A_{\psi^{I}}(\cdot, \cdot)$ is the bilinear form on $\left(H_{D}^{1}(\Omega)\right)^{2}$ defined by (2.9) with $\psi$ replaced by $\psi^{I}$.

Problem 3.2 is a discrete problem corresponding to Problem 2.1. The existence and uniqueness of the solution to this problem is established in the following theorem. 
Theorem 3.3. There exists a unique solution to Problem 3.2.

Proof. From (2.9) and (2.11) we have

$$
A_{\psi^{I}}\left(v_{h}, v_{h}\right)=\left\|v_{h}\right\|_{1, \psi^{I}}^{2} \quad \forall v_{h} \in S_{h},
$$

i.e., $A_{\psi^{I}}(\cdot, \cdot)$ is coercive on $S_{h} \times S_{h}$. Therefore, the existence and uniqueness of the solution to Problem 3.2 follows from the well-known Lax-Milgram theorem.

We comment that (3.15) also implies the stability of the method.

Let $u_{h}=\sum_{i=1}^{N^{\prime}} u_{i} \phi_{i}$ and $v_{h}=\phi_{j}\left(j=1,2, \ldots, N^{\prime}\right)$. Substituting these into (3.15) we obtain a linear algebraic system of $\left\{u_{i}\right\}_{1}^{N^{\prime}}$. The system matrix is symmetric because from $(2.9)$ we see that $A_{\psi^{I}}(\cdot, \cdot)$ is symmetric. Equality (3.15) implies that the system matrix is also positive definite.

We comment that the system matrix assembly of the method involves the evaluation of the qualities $\boldsymbol{f}_{\phi_{i}, \boldsymbol{a}^{I}}=$ $\nabla \phi_{i}-\boldsymbol{a}^{I} \phi_{i}$ for $i=1,2, \ldots, N$. For each $i, \nabla \phi_{i}$ is not explicitly given, but it is not difficult to obtain an analytic expression for $\nabla \phi_{i}$ from (3.10). Since, in practice, a numerical quadrature rule on each triangle is normally used for the evaluation of the integrals in the system matrix, we can also evaluate the numerical values of $\nabla \phi_{i}$ using (3.10). Moreover, we may simply use $\boldsymbol{p}_{i}$ as an approximation of $\boldsymbol{f}_{\phi_{i}, \boldsymbol{a}^{I}}$. This is because by Theorem 3.2, $\boldsymbol{p}_{i}=\boldsymbol{f}_{\phi_{i}, \boldsymbol{a}^{I}}$ at all vertices of $T_{h}$. Thus, the matrix assembly of this method should be reasonably inexpensive, although it need more floating point operations than the conventional Scharfetter-Gummel box method (cf. [11]).

\section{Convergence of the finite element solution}

In the previous section we constructed a conforming finite element space. Using that space a Bubnov-Galerkin finite element problem was formulated which was shown to be uniquely solvable. In this section we show that the errors of the solution to Problem 3.1 in the energy norm and its associated flux in the $\boldsymbol{L}^{2}$-norms are of order $h$.

Before discussing the convergence of the approximate solution we first consider the interpolation accuracy of the space $S_{h}$. Unlike the standard piecewise linear space, this space is based on a constant approximation to the flux of a given function on any segment in an element, and yields an exponential approximation to the function in each element. The following theorem gives the local error bounds for the $S_{h}$-interpolant and its associated flux of a smooth function.

Theorem 4.1. Let $w_{I}$ be the $S_{h}$-interpolant of a sufficiently smooth function $w$ and let $\boldsymbol{f}_{w, \boldsymbol{a}^{I}}$ and $\boldsymbol{f}_{w_{I}, \boldsymbol{a}^{I}}$ be the associated fluxes defined by (2.5). Then there exist positive constants $C_{1}$ and $C_{2}$, independent of $h$ and $w$, such that, for any $t \in T_{h}$,

$$
\begin{aligned}
& \left\|\boldsymbol{f}_{w, \boldsymbol{a}^{I}}-\boldsymbol{f}_{w_{I}, \boldsymbol{a}^{I}}\right\|_{\infty, t} \leq C_{1} h\left(\left|\boldsymbol{f}_{w, \boldsymbol{a}}\right|_{1, \infty, t}+|w|_{1, \infty, t}|\boldsymbol{a}|_{1, \infty, t}\right), \\
& \| w-\left.w_{I}\right|_{\infty, t} \leq C_{2} h^{2}\left(\left|\boldsymbol{f}_{w, \boldsymbol{a}}\right|_{1, \infty, t}+|w|_{1, \infty, t}|\boldsymbol{a}|_{1, \infty, t}\right)
\end{aligned}
$$

Proof. Let $C>0$ be a generic constant, independent of $h$ and $w$. Now, if $\boldsymbol{f}_{w, \boldsymbol{a}^{I}}=\left(d_{1}, d_{2}\right)$ with constants $d_{1}$ and $d_{2}$, then, for any $t \in T_{h}$ with vertices $x_{i}, x_{j}$ and $x_{k}$ and any $x \in t$ we see that $\left(d_{1}, d_{2}\right)$ and $w(x)$ satisfy

$$
\boldsymbol{f}_{w, \boldsymbol{a}^{I}} \cdot \boldsymbol{e}_{m} \equiv\left(d_{1}, d_{2}\right) \cdot \boldsymbol{e}_{m}=\frac{1}{\left|l_{m}\right|}\left[B\left(a_{m}\left|l_{m}\right|\right) w(x)-B\left(-a_{m}\left|l_{m}\right|\right) w\left(x_{m}\right)\right]
$$

for all $x \in l_{m}$ and $m=i, j, k$. From this we have

$$
D(x)\left(\begin{array}{c}
d_{1} \\
d_{2} \\
w(x)
\end{array}\right)=\left(\begin{array}{c}
-B\left(-a_{i}\left|l_{i}\right|\right) w\left(x_{i}\right) \\
-B\left(-a_{j}\left|l_{j}\right|\right) w\left(x_{j}\right) \\
-B\left(-a_{k}\left|l_{k}\right|\right) w\left(x_{k}\right)
\end{array}\right)
$$


where $B(\cdot)$ is the Bernoulli function defined in (3.3) and $D(x)$ is the matrix defined by (3.5). This matrix equality shows that $w \in S_{h}$ in the case that $\boldsymbol{f}_{w, \boldsymbol{a}^{I}}$ is a constant vector, and thus the mapping $\boldsymbol{f}_{w, \boldsymbol{a}^{I}} \mapsto \boldsymbol{p}_{w_{I}}$ preserves constants, where $\boldsymbol{p}_{w_{I}}$ denotes the auxiliary flux associated with $w_{I}$. From Theorem 3.2 we see that the mapping $\boldsymbol{p}_{w_{I}} \mapsto \boldsymbol{f}_{w_{I}} \boldsymbol{a}^{I}$ also preserves constants, because the nodal values of both qualities are equal at the vertices of $T_{h}$. So, using a standard argument (cf., for example, [5], Theorem 3.1.4) we have that

$$
\begin{aligned}
\left\|\boldsymbol{f}_{w, \boldsymbol{a}^{I}}-\boldsymbol{f}_{w_{I}, \boldsymbol{a}^{I}}\right\|_{\infty, t} & \leq C h\left|\boldsymbol{f}_{w, \boldsymbol{a}^{I}}\right|_{1, \infty, t} \\
& \leq C h\left(\left|\boldsymbol{f}_{w, \boldsymbol{a}}\right|_{1, \infty, t}+\left|w\left(\boldsymbol{a}-\boldsymbol{a}^{I}\right)\right|_{1, \infty, t}\right) \\
& \leq C h\left(\left|\boldsymbol{f}_{w, \boldsymbol{a}}\right|_{1, \infty, t}+|w|_{1, \infty, t}|\boldsymbol{a}|_{1, \infty, t}\right),
\end{aligned}
$$

since $\boldsymbol{a}^{I}$ is constant on $t$. This proves (4.1).

Using the notation defined in Section 3 (cf. Fig. 3.1), we now show (4.2). Let $x_{i}$ be a vertex of $t \in T_{h}$. We have, for any $x \in \bar{t}$,

$$
\begin{aligned}
\frac{d E(y)}{d \boldsymbol{e}_{i}}-a_{i} E(y) & =\left(\boldsymbol{f}_{w, \boldsymbol{a}^{I}}-\boldsymbol{f}_{w_{I}, \boldsymbol{a}^{I}}\right) \cdot \boldsymbol{e}_{i}, \quad \forall y \in l_{i} \\
E\left(x_{i}\right) & =0,
\end{aligned}
$$

where $E=w-w_{I}$. The solution of this initial value problem at $y=x$ is

$$
E(x)=e^{a_{i}\left|l_{i}\right|}\left[\int_{0}^{\left|l_{i}\right|}\left(\boldsymbol{f}_{w, \boldsymbol{a}^{I}}-\boldsymbol{f}_{w_{I}, \boldsymbol{a}^{I}}\right) \cdot \boldsymbol{e}_{i} e^{-a_{i} s} d s+K\right]
$$

where $K$ is a constant to be determined. Since $E\left(x_{i}\right)=0$ we have $K=0$, and thus from the above we obtain

$$
\begin{aligned}
|E(x)| & \leq e^{a_{i}\left|l_{i}\right|}\left\|\boldsymbol{f}_{w, \boldsymbol{a}^{I}}-\boldsymbol{f}_{w_{I}, \boldsymbol{a}^{I}}\right\|_{\infty, t} \int_{0}^{\left|l_{i}\right|} e^{-a_{i} s} d s \\
& =e^{a_{i}\left|l_{i}\right|}\left\|\boldsymbol{f}_{w, \boldsymbol{a}^{I}}-\boldsymbol{f}_{w_{I}, \boldsymbol{a}^{I}}\right\|_{\infty, t} \frac{1-e^{-a_{i}\left|l_{i}\right|}}{a_{i}} \\
& =\| \boldsymbol{f}_{w, \boldsymbol{a}^{I}}-\boldsymbol{f}_{w_{I}, \boldsymbol{a}^{I} \|_{\infty, t} B^{-1}\left(a_{i}\left|l_{i}\right|\right)\left|l_{i}\right|}
\end{aligned}
$$

where $B(z)$ is the Bernoulli function defined by (3.3). Note that this inequality also covers the case that $a_{i}=0$, because $B(0)=1$. Combining this inequality and (4.1) we obtain (4.2).

We now consider the convergence of the finite element solution. On $\left(W^{1, \infty}(\Omega)\right)^{2}$ we introduce the functional $|\cdot|_{1, \infty, \psi^{I}, h}$ by defining, for any $\boldsymbol{p} \in\left(W^{1, \infty}(\Omega)\right)^{2}$,

$$
|\boldsymbol{p}|_{1, \infty, \psi^{I}, h}=\left(\sum_{t \in T_{h}} \int_{t} e^{-\psi^{I}} d x|\boldsymbol{p}|_{1, \infty, t}^{2}\right)^{1 / 2} .
$$

Obviously $|\cdot|_{1, \infty, \psi^{I}, h}$ is a seminorm on $\left(W^{1, \infty}(\Omega)\right)^{2}$. Using this seminorm we have the following theorem which establishes the convergence of the finite element approximations $u_{h}$ and $\boldsymbol{f}_{u_{h}}, \boldsymbol{a}^{I}$ to the exact solutions $u$ and $\boldsymbol{f}_{u, \boldsymbol{a}}$.

Theorem 4.2. Let $u$ and $u_{h}$ be the solutions to Problem 2.1 and Problem 3.1 respectively. Then there exists a constant $C>0$, independent of $h, u$ and $\psi$, such that

$$
\begin{aligned}
\left\|u-u_{h}\right\|_{1, \psi^{I}} & \leq C h\left(\left|\boldsymbol{f}_{u, \boldsymbol{a}}\right|_{1, \infty, \psi^{I}, h}+|u|_{1, \infty}|\boldsymbol{a}|_{1, \infty, \psi^{I}, h}\right), \\
\left\|\boldsymbol{f}_{u, \boldsymbol{a}}-\boldsymbol{f}_{u_{h}, \boldsymbol{a}^{I}}\right\|_{0, \psi^{I}} & \leq C h\left(\left|\boldsymbol{f}_{u, \boldsymbol{a}}\right|_{1, \infty, \psi^{I}, h}+\|u\|_{1, \infty}|\boldsymbol{a}|_{1, \infty, \psi^{I}, h}\right) .
\end{aligned}
$$


Proof. Let $u_{I} \in S_{h}$ be the $S_{h}$-interpolant of $u$. From (2.8) and (3.14) we have

$$
A_{\psi^{I}}\left(u_{h}, v_{h}\right)=A_{\psi^{I}}\left(u, v_{h}\right), \quad \forall v_{h} \in S_{h} .
$$

Subtracting $A_{\psi^{I}}\left(u_{I}, v_{h}\right)$ from both sides of this equality and using (2.11) we obtain, for any $v_{h} \in S_{h}$,

$$
\begin{aligned}
& A_{\psi^{I}}\left(u_{h}-u_{I}, v_{h}\right)=A_{\psi^{I}}\left(u-u_{I}, v_{h}\right) \\
& =\left(\nabla\left(u-u_{I}\right)-\boldsymbol{a}^{I}\left(u-u_{I}\right), \nabla v_{h}-\boldsymbol{a}^{I} v_{h}\right)_{\psi^{I}}+\left(G\left(u-u_{I}\right), v_{h}\right)_{\psi^{I}} \\
& =\left(\boldsymbol{f}_{u, \boldsymbol{a}^{I}}-\boldsymbol{f}_{u_{I}, \boldsymbol{a}^{I}}, \boldsymbol{f}_{v_{h}, \boldsymbol{a}^{I}}\right)_{\psi^{I}}+\left(G\left(u-u_{I}\right), v_{h}\right)_{\psi^{I}} \\
& \leq\left\|\boldsymbol{f}_{u, \boldsymbol{a}^{I}}-\boldsymbol{f}_{u_{I}, \boldsymbol{a}^{I}}\right\|_{0, \psi^{I}}\left\|\boldsymbol{f}_{v_{h}, \boldsymbol{a}^{I}}\right\|_{0, \psi^{I}}+\left(G\left(u-u_{I}\right), u-u_{I}\right)_{\psi^{I}}^{1 / 2}\left(G v_{h}, v_{h}\right)_{\psi^{I}}^{1 / 2} \\
& \leq C\left(h+h^{2}\|G\|_{0, \infty}^{1 / 2}\right) \\
& \cdot\left(\sum_{t \in T_{h}} \int_{t} e^{-\psi^{I}} d x\left(\left|\boldsymbol{f}_{u}\right|_{1, \infty, t}+|u|_{1, \infty, t}|\boldsymbol{a}|_{1, \infty, t}\right)^{2}\right)^{1 / 2}\left\|v_{h}\right\|_{1, \psi^{I}} \\
& \leq C h\left(\left|\boldsymbol{f}_{u}\right|_{1, \infty, \psi^{I}, h}+|u|_{1, \infty}|\boldsymbol{a}|_{1, \infty, \psi^{I}, h}\right)|| v_{h} \|_{1, \psi} .
\end{aligned}
$$

In the above we used (4.1), (4.2), (4.4) and the Cauchy-Schwarz inequality. Letting $v_{h}=u_{h}-u_{I}$, we obtain from (3.15) and the above inequality

$$
\| u_{h}-\left.u_{I}\right|_{1, \psi^{I}} \leq C h\left(\left|\boldsymbol{f}_{u, \boldsymbol{a}}\right|_{1, \infty, \psi^{I}, h}+|u|_{1, \infty}|\boldsymbol{a}|_{1, \infty, \psi^{I}, h}\right) .
$$

Analogously to the derivation of the about inequality, we have from (2.10), (4.1) and (4.2)

$$
\left\|u-u_{I}\right\|_{1, \psi^{I}}^{2}=A_{\psi^{I}}\left(u-u_{I}, u-u_{I}\right) \leq C h\left(\left|\boldsymbol{f}_{u, \boldsymbol{a}}\right|_{1, \infty, \psi, h}+|u|_{1, \infty}|\boldsymbol{a}|_{1, \infty, \psi^{I}, h}\right)\left\|u-u_{I}\right\|_{1, \psi^{I}},
$$

and so

$$
\| u-\left.u_{I}\right|_{1, \psi^{I}} \leq C h\left(\left|\boldsymbol{f}_{u, \boldsymbol{a}}\right|_{1, \infty, \psi, h}+|u|_{1, \infty}|\boldsymbol{a}|_{1, \infty, \psi^{I}, h}\right) .
$$

Therefore (4.5) follows from this, the triangular inequality and (4.7).

We now prove (4.6). Using (2.11) we see that (4.5) implies that

$$
\| \boldsymbol{f}_{u, \boldsymbol{a}^{I}}-\left.\boldsymbol{f}_{u_{h}, \boldsymbol{a}^{I}}\right|_{0, \psi^{I}} \leq C h\left(\left|\boldsymbol{f}_{u, \boldsymbol{a}}\right|_{1, \infty, \psi, h}+|u|_{1, \infty}|\boldsymbol{a}|_{1, \infty, \psi^{I}, h}\right) .
$$

By the triangle inequality and (4.1), we get

$$
\begin{aligned}
\left\|\boldsymbol{f}_{u, \boldsymbol{a}}-\boldsymbol{f}_{u_{h}, \boldsymbol{a}^{I}}\right\|_{0, \psi^{I}} \leq & \left\|\boldsymbol{f}_{u, \boldsymbol{a}^{I}}-\boldsymbol{f}_{u_{h}, \boldsymbol{a}^{I}}\right\|_{0, \psi^{I}}+\left\|u\left(\boldsymbol{a}-\boldsymbol{a}^{I}\right)\right\|_{0, \psi^{I}} \\
\leq & \operatorname{Ch}\left(\left|\boldsymbol{f}_{u, \boldsymbol{a}}\right|_{1, \infty, \psi, h}+|u|_{1, \infty}|\boldsymbol{a}|_{1, \infty, \psi^{I}, h}\right) \\
& +C h\|u\|_{\infty}|\boldsymbol{a}|_{1, \infty, \psi^{I}, h} \\
\leq & \operatorname{Ch}\left(\left|\boldsymbol{f}_{u, \boldsymbol{a}}\right|_{1, \infty, \psi, h}+\|u\|_{1, \infty}|\boldsymbol{a}|_{1, \infty, \psi^{I}, h}\right)
\end{aligned}
$$

proving (4.6).

Theorem 4.2 shows that the solution of Problem 3.1 and its associated flux converge to the exact ones with the convergence rate of $O(h)$ order. The error bounds depend only on $\|u\|_{1, \infty}$ and the weighted first order seminorms of the exact flux and the coefficient function of the convection term. This is in contrast to the standard piecewise linear finite element method in which the error bound depends on $\|u\|_{2}$. Unlike most of the previous work the variable used in (4.5) is the electron or hole concentration rather than one of the Slotboom variables which are physically less interesting. 


\section{Evaluation of terminal CURRENTs}

We now consider the evaluation of the ohmic contact currents, which is often the final goal of device simulation. This discussion is similar to that in [6]. For simplicity, we restrict our attention to a device with a finite number of ohmic contacts, and so $\partial \Omega_{D}$ is a finite set of separated contacts. We assume that the mesh $T_{h}$ is such that the end-points of any contact are mesh nodes of $T_{h}$. From the definition of ohmic contacts we know that the potential drop within a contact is negligible (cf. [17], p.304). Thus $\psi$ is constant on each ohmic contact. Let $V_{h}:=\operatorname{span}\left\{\phi_{i}\right\}_{1}^{N} \subset C^{0}(\bar{\Omega}) \cap H^{1}(\Omega)$. Obviously, if $v \in V_{h}$ and $\left.v\right|_{\partial \Omega_{D}}=0$, then $v \in S_{h}$. For any $c \in \partial \Omega_{D}$, we choose $\phi_{c} \in V_{h}$ satisfying

$$
\phi_{c}(x)= \begin{cases}e^{\psi_{c}} & x \in c \\ 0 & x \in \partial \Omega_{D} \backslash c,\end{cases}
$$

where $\psi_{c}$ denotes the (constant) value of $\psi$ on $c$. (The value of the piecewise linear interpolant $\psi^{I}$ of $\psi$ on $c$ is also equal to $\psi_{c}$.) Taking $G=0$ in (2.4), multiplying by $e^{-\psi^{I}} \phi_{c}$ and integrating by parts we have

$$
-\int_{c} \boldsymbol{f}_{u, \boldsymbol{a}} \cdot \boldsymbol{n} d s+\left(\boldsymbol{f}_{u, \boldsymbol{a}}, \boldsymbol{f}_{\phi_{c}, \boldsymbol{a}^{I}}\right)_{\psi^{I}}=\left(F, \phi_{c}\right)_{\psi^{I}} .
$$

Thus the outflow current through $c$ is

$$
J_{c}:=\int_{c} \boldsymbol{f}_{u, \boldsymbol{a}} \cdot \boldsymbol{n} d s=\left(\boldsymbol{f}_{u, \boldsymbol{a}}, \boldsymbol{f}_{\phi_{c}, \boldsymbol{a}^{I}}\right)_{\psi^{I}}-\left(F, \phi_{c}\right)_{\psi^{I}} .
$$

Replacing $\boldsymbol{f}_{u, \boldsymbol{a}}$ by the approximate flux $\boldsymbol{f}_{u_{h}, \boldsymbol{a}^{I}}$ we obtain the following approximate outflow current through $c$

$$
J_{c, h}:=\left(\boldsymbol{f}_{u_{h}, \boldsymbol{a}^{I}}, \boldsymbol{f}_{\phi_{c}, \boldsymbol{a}^{I}}\right)_{\psi^{I}}-\left(F, \phi_{c}\right)_{\psi^{I}} .
$$

The convergence and the conservation of the computed ohmic contact currents are established in the following theorem.

Theorem 5.1. Let $J_{c}$ and $J_{c, h}$ be respectively the exact and the computed outflow currents through $c \in \partial \Omega_{D}$. Then, there exists a constant $C>0$, independent of $h, \psi$ and $u$, such that

$$
\left|J_{c}-J_{c, h}\right| \leq C h\left(\left|\boldsymbol{f}_{u, \boldsymbol{a}}\right|_{1, \infty, \psi^{I}, h}+\|u\|_{1, \infty}|\boldsymbol{a}|_{1, \infty, \psi^{I}, h}\right)\left\|\phi_{c}\right\|_{1, \psi^{I}}
$$

Furthermore

$$
\sum_{c \in \partial \Omega_{D}} J_{c, h}=-\int_{\Omega} F d x
$$

Proof. Let $C$ denote a generic positive constant, independent of $h, \psi$ and $u$. From (5.2), (5.3) and (4.6) we obtain

$$
\begin{aligned}
\left|J_{c}-J_{c, h}\right| & =\mid\left(\boldsymbol{f}_{u, \boldsymbol{a}}-\boldsymbol{f}_{u_{h}, \boldsymbol{a}^{I}}, \boldsymbol{f}_{\left.\phi_{c}, \boldsymbol{a}^{I}\right)_{\psi^{I}} \mid}\right. \\
& \leq\left\|\boldsymbol{f}_{u, \boldsymbol{a}}-\boldsymbol{f}_{u_{h}, \boldsymbol{a}^{I}}\right\|_{0, \psi^{I}}\left\|\boldsymbol{f}_{\phi_{c}}\right\|_{0, \psi} \\
& \leq \operatorname{Ch}\left(\left|\boldsymbol{f}_{u}\right|_{1, \infty, \psi^{I}, h}+\|u\|_{1, \infty}|\boldsymbol{a}|_{1, \infty, \psi^{I}, h}\right)\left\|\phi_{c}\right\|_{1, \psi^{I}},
\end{aligned}
$$

proving (5.4).

To prove (5.5) we first notice

$$
\boldsymbol{f}_{e^{\psi^{I}}, \boldsymbol{a}^{I}}=\nabla e^{\psi^{I}}-e^{\psi^{I}} \nabla \psi^{I} \equiv 0 .
$$


Let $\phi=\sum_{c \in \partial \Omega_{D}} \phi_{c}$. Summing (5.3) over all the contacts and using the above identity we have

$$
\begin{aligned}
\sum_{c \in \partial \Omega_{D}} J_{c, h} & =\left(\boldsymbol{f}_{u_{h}, \boldsymbol{a}^{I}}, \sum_{c \in \partial \Omega_{D}} \boldsymbol{f}_{\phi_{c}, \boldsymbol{a}^{I}}\right)_{\psi^{I}}-\left(F, \sum_{c \in \partial \Omega_{D}} \phi_{c}\right)_{\psi_{I}} \\
& =\left(\boldsymbol{f}_{u_{h}, \boldsymbol{a}^{I}}, \boldsymbol{f}_{\phi, \boldsymbol{a}^{I}}\right)_{\psi^{I}}-(F, \phi)_{\psi^{I}} \\
& =\left(\boldsymbol{f}_{u_{h}, \boldsymbol{a}^{I}}, \boldsymbol{f}_{\phi, \boldsymbol{a}^{I}}-\boldsymbol{f}_{e^{\psi^{I}}, \boldsymbol{a}^{I}}\right)_{\psi^{I}}-\left(F, \phi-e^{\psi^{I}}\right)_{\psi^{I}}-\left(F, e^{\psi^{I}}\right)_{\psi^{I}} \\
& =A_{\psi^{I}}\left(u_{h}, \phi-e^{\psi^{I}}\right)-\left(F, \phi-e^{\psi^{I}}\right)_{\psi^{I}}-(F, 1)
\end{aligned}
$$

since $\phi-e^{\psi^{I}} \in H_{D}^{1}(\Omega)$. It remains to show that $\phi-e^{\psi^{I}} \in S_{h}$. Then (5.5) follows from the above equality and (3.14). Since $\phi \in V_{h}$, if we can show that $e^{\psi^{I}} \in V_{h}$, then $\phi-e^{\psi^{I}} \in S_{h}$, because from (5.1) we have $\left.\left(\phi-e^{\psi^{I}}\right)\right|_{\partial \Omega_{D}}=0$. In fact, from the proof of Theorem 4.1 we know that for any $t \in T_{h}$ and any $x \in t$, $\left(0,0, e^{\psi^{I}(x)}\right)$ satisfies (4.3) with $w$ replaced by $e^{\psi^{I}}$. This is because $\boldsymbol{f}_{e^{\psi^{I}}} \boldsymbol{a}^{I} \equiv 0$ on $\Omega$. Thus, $e^{\psi^{I}} \in V_{h}$. This completes the proof.

The total terminal current $J_{c, h}^{\text {tot }}$ flowing out of $c$ is equal to the sum of the electron and hole currents, i.e.

$$
J_{c, h}^{t o t}=J_{c, h}^{n}+J_{c, h}^{p}
$$

where $J_{c, h}^{n}$ and $J_{c, h}^{p}$ denote respectively the computed electron and hole currents flowing out of $c$ by (5.3). From Theorem 5.1 we have the following corollary.

Corollary 5.1. The computed total current flowing out of $\partial \Omega_{D}$ is conservative, i.e.

$$
\sum_{c \in \partial \Omega_{D}} J_{c, h}^{t o t}=0
$$

Proof. Noticing that the right-hand sides of (2.2) and (2.3) are respectively $R$ and $-R,(5.7)$ follows from (5.5) and (5.6).

\section{Conclusion}

In this paper we presented a novel exponentially fitted finite element method with triangular elements for the semiconductor continuity equations. The continuous problem was formulated as a variational problem using the weighted inner product, and a Bubnov-Galerkin finite element method with a set of special piecewise exponential basis functions was then proposed to approximate the variational problem. Each of these basis functions and the auxiliary flux associated with it are uniquely determined by a set of two-point boundary problems. The method was proved to be stable and error estimates for the approximate solution and the associated flux were given. These error bounds depend only on $\|u\|_{1, \infty}$, and on the first order seminorms of the exact flux and the coefficient function of the convection term. A method was also proposed for the evaluation of terminal currents and it was shown that the computed terminal currents are convergent and conservative.

The author is grateful to the referees for several helpful observations. The support of the Australian Research Council is also gratefully acknowledged. 


\section{REFERENCES}

[1] D.N. de G. Allen, R.V. Southwell, Relaxation methods applied to determine the motion, in two dimensions, of a viscous fluid past a fixed cylinder. Quart. J. Mech. Appl. Math. 8 (1955) 129-145.

[2] R.E. Bank, J.F. Bürgler, W. Fichtner, R.K. Smith, Some upwinding techniques for finite element approximations of convectiondiffusion equations. Numer. Math. 58 (1990) 185-202.

[3] F. Brezzi, P. Marini, P. Pietra, Two-dimensional exponentially fitting and applications to semiconductor device equations. SIAM J. Numer. Anal. 26 (1989) 1342-1355.

[4] E. Buturla, P. Cottrell, B.M. Grossman, K.A. Salsburg, Finite Element Analysis of Semiconductor Devices: The FIELDAY Program. IBM J. Res. Develop. 25, (1981) 218-231.

[5] P.G. Ciarlet, The finite element method for elliptic problems. North-Holland, Amsterdam (1978).

[6] C.J. Fitzsimons, J.J.H. Miller, S. Wang, C.H. Wu, Hexahedral finite elements for the stationary semiconductor device equations. Comp. Meth. Appl. Mech. Engrg. 84 (1990) 43-57.

[7] H.K. Gummel, A self-consistent iterative scheme for one-dimensional Steady State Transistor Calculation. IEEE Trans. Elec. Dev. 11 (1964) 455-465.

[8] P.A. Markowich, M. Zlámal, Inverse-Average-Type Finite Element Discretisations of Selfadjoint Second-Order Elliptic Problems. Math. Comp. 51 (1988) 431-449.

[9] B.J. McCartin, Discretization of the Semiconductor Device Equations. From New Problems and New Solutions for Device and Process Modelling. J.J.H. Miller Ed. Boole Press, Dublin (1985).

[10] J.J.H. Miller and S. Wang, A Triangular Mixed Finite Element Method for the Stationary Semiconductor Device Equations. RAIRO Modél. Math. Anal. Numér. 25 (1991) 441-463.

[11] J.J.H. Miller and S. Wang, An analysis of the Scharfetter-Gummel box method for the stationary semiconductor device equations. RAIRO Modél. Math. Anal. Numér. 28 (1994) 123-140.

[12] J.J.H. Miller and S. Wang, A tetrahedral mixed finite element method for the stationary semiconductor continuity equations. SIAM J. Numer. Anal. 31 (1994) 196-216.

[13] M.S. Mock, Analysis of a Discretization Algorithm for Stationary Continuity Equations in Semiconductor Device Models. COMPEL 2 (1983) 117-139.

[14] D. Scharfetter and H.K. Gummel, Large-signal analysis of a silicon read diode oscillator. IEEE Trans. Elec. Dev. 16 (1969) 64-77.

[15] M. Sever, Discretization of time-dependent continuity equations. Proceedings of the 6th International NASECODE Conference. J.J.H. Miller Ed. Boole Press, Dublin (1988) 71-83.

[16] J.W. Slotboom, Iterative Scheme for 1- and 2-Dimensional D.C.-Transistors. IEEE Trans. Elect. Dev. 24 (1977) 1123-1125.

[17] S.M. Sze, The physics of semiconductor devices, 2nd ed. John Wiley \& Sons, New York (1981)

[18] W.V. Van Roosbroeck, Theory of Flow of Electrons and Holes in Germanium and Other Semiconductors. Bell Syst. Tech. J. 29 (1950) 560-607. 